\section{Aachener Allergiezentrum als CAC zertifiziert}

Allergien sind eine multifaktorielle Erkrankung, die in den vergangenen Jahren kontinuierlich zugenommen haben. Die wichtige interdisziplinäre Zusammenarbeit leisten zertifizierte Comprehensive Allergy Center (CAC). An der Uniklinik RWTH Aachen wurde nun das sechste CAC in Deutschland zertifiziert.

D. as Aachener Comprehensive Allergy Center (ACAC) ist eine zentrale Anlaufstelle zum Thema Allergie - nicht nur für Betroffene, auch für niedergelassene Ärzte. Allergiepatienten bietet es durch die Bündelung der universitären Kompetenz eine Versorgung auf höchstem Niveau. „Da die Zahl der Allergiker stetig zunimmt, wird eine umfassende Versorgung von Betroffenen immer wichtiger. Viele unterschätzen Allergien, dabei können sie gesundheitsgefährdend und im schlimmsten Fall lebensbedrohend sein", erklärt Prof. Dr. Jens Malte Baron, Sprecher des ACAC. Patienten profitieren auch von dem engen
Netzwerk verschiedener Fachabteilungen mit niedergelassenen Ärzten. Zudem besteht eine direkte Zusammenarbeit mit externen Kooperationspartnern und Einweisern in Aachen und Umgebung.

\section{Gelebte Kooperation in Aachen}

An der Uniklinik RWTH Aachen besteht die interdisziplinäre Zusammenarbeit im Bereich der Allergologie zwischen insgesamt elf Kliniken und Instituten, darunter die Klinik für Dermatologie und Allergologie und die Sektion Pneumologie an der Klinik für Kardiologie, Pneumologie, Angiologie und Internistische Intensivmedizin (Medizinische Klinik I),

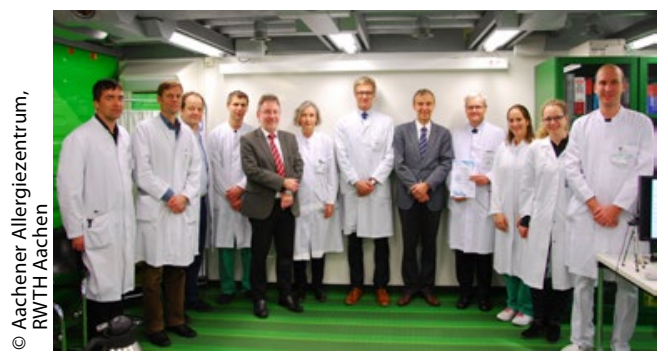

Geschafft! Das Aachener Allergiezentrum ist jetzt als CAC zertifiziert.

die mit Prof. Dr. Baron und Prof. Dr. Dreher die Sprecher des Zentrums stellen. Mit der Zertifizierung garantiert das ACAC eine Versorgung auf höchstem Niveau. Die Gutachter Prof. Dr. Thomas Werfel und Prof. Dr. Randolf Brehler lobten bei ihrer Begutachtung des Zentrums die bemerkenswert enge, interdisziplinäre Zusammenarbeit. Dabei sei besonders die bereits seit drei Jahren bestehende Allergologiesprechstunde hervorzuheben, die von einer gelebten Kooperation zeuge und eine hoch kompetente Versorgung der Patienten ermögliche.

\section{Allergologie im Kloster}

Zum 13. Mal findet in diesem Jahr das interdisziplinäre Symposium "Allergologie im Kloster" statt - am 19. und 20. Mai in den historischen Gemäuern des Kloster Eberbach in Eltville, Rheingau.

Tagungsleiter Prof. Dr. Ludger Klimek lädt alle Interessierten herzlich ein: „Porta patet, cor magis - die Tür steht offen, mehr noch das Herz! Seit einigen Jahren dürfen wir Allergologen uns jeweils im Wonnemonat Mai diesem Wahlspruch folgend im Rheingau zusammenfinden, um aktuelle Forschungsergebnisse auszutauschen und Weiterbildungsreferate auf hohem Niveau zu verschiedenen Themen zu hören."

Hier einige Highlights aus dem Hauptprogramm:

- 45 Jahre Kinderallergologie - Fehler, Irrtümer,

Enttäuschungen. Was bleibt? U. Wahn

_ Das Anaphylaxie-Register: Erkenntnisse für Patienten, Ärzte und die Wissenschaft. M. Worm

_ Was bedeutet der Klimawandel für Allergiker und Allergologen. K.-C. Bergmann

_ Das atopische Ekzem: Eine aktuelle Bestandsaufnahme. J. Ring

_ Diäten bei Nahrungsmittelallergien und -intoleranzen:

Therapie oder Modeerscheinung. I. Reese

_ Das ASS-Intoleranz-Syndrom: Polyposis nasi, Asthma und mehr. L. Klimek

Darüber hinaus wird es praktische Workshops geben, z. B.:

_ Workshop I: Der allergologische Notfall / Anaphylaxie: Wie gehe ich in der Praxis vor?

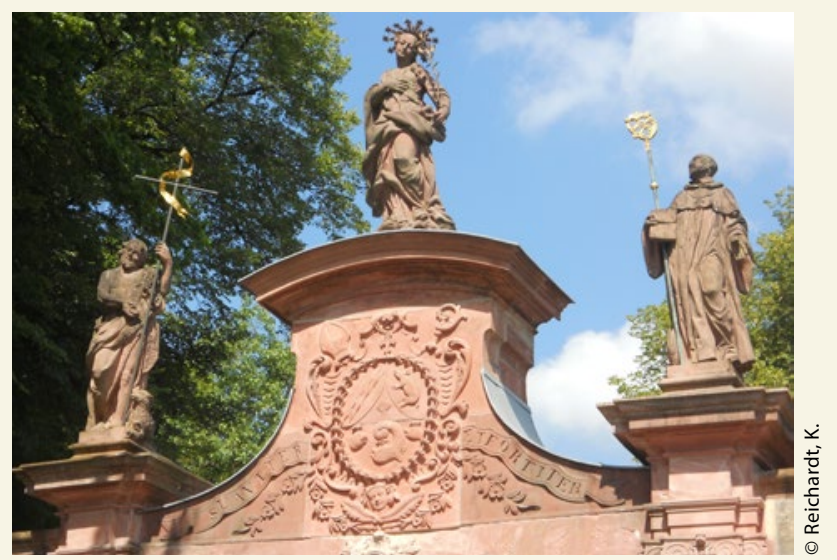

_ Workshop II: Akupunkturkurs: Grundlagen und HNO-spezifische Behandlungsstrategien mit praktischen Übungen _ Workshop III: FeNO-Messungen in der Praxis: Praktische Übungen und Hands-on Workshop
Anmeldung und Information
Katja Reichardt
Zentrum für Rhinologie und Allergologie
An den Quellen 10, 65183 Wiesbaden
Tel.: (06 11) 308608290
Fax: (06 11) 308608295
E-Mail: info@allergologie-im-kloster.org
Internet: www.allergologie-im-kloster.org 